\title{
Roles for Drosophila mushroom body neurons in olfactory learning and memory
}

\author{
David-Benjamin G. Akalal, ${ }^{1,4}$ Curtis F. Wilson, ${ }^{1}$ Lin Zong, ${ }^{1}$ Nobuaki K. Tanaka, ${ }^{2}$ \\ Kei Ito, ${ }^{2}$ and Ronald L. Davis ${ }^{1,3,4}$ \\ ${ }^{1}$ Department of Molecular and Cellular Biology, Baylor College of Medicine, Houston, Texas 77030, USA; ${ }^{2}$ Institute of Molecular \\ and Cellular Biosciences, University of Tokyo, Bunkyo-ku, Tokyo 113-0032, Japan; ${ }^{3}$ Menninger Department of Psychiatry and \\ Behavioral Sciences, Baylor College of Medicine, Houston, Texas 77030, USA
}

\begin{abstract}
Olfactory learning assays in Drosophila have revealed that distinct brain structures known as mushroom bodies (MBs) are critical for the associative learning and memory of olfactory stimuli. However, the precise roles of the different neurons comprising the MBs are still under debate. The confusion surrounding the roles of the different neurons may be due, in part, to the use of different odors as conditioned stimuli in previous studies. We investigated the requirements for the different $M B$ neurons, specifically the $\boldsymbol{\alpha} / \boldsymbol{\beta}$ versus the $\boldsymbol{\gamma}$ neurons, and whether olfactory learning is supported by different subsets of $\mathrm{MB}$ neurons irrespective of the odors used as conditioned stimuli. We expressed the rutabaga (rut)-encoded adenylyl cyclase in either the $\boldsymbol{\gamma}$ or $\boldsymbol{\alpha} / \boldsymbol{\beta}$ neurons and examined the effects on restoring olfactory associative learning and memory of rut mutant flies. We also expressed a temperature-sensitive shibire (shi) transgene in these neuron sets and examined the effects of disrupting synaptic vesicle recycling on Drosophila olfactory learning. Our results indicate that although we did not detect odor-pair-specific learning using GAL4 drivers that primarily express in $\gamma$ neurons, expression of the transgenes in a subset of $\boldsymbol{\alpha} / \boldsymbol{\beta}$ neurons resulted in both odor-pair-specific rescue of the rut defect as well as odor-pair-specific disruption of learning using shitsl.
\end{abstract}

Numerous studies indicate that the Drosophila mushroom bodies (MBs), a paired neuropil structure in the central brain, are critical for associative learning and memory (for review, see Roman and Davis 2001; Waddell and Quinn 2001; Dubnau et al. 2003; Heisenberg 2003; Davis 2005). The MBs are composed of three distinct classes of neurons-alpha/beta $(\alpha / \beta)$, alpha'/beta' $\left(\alpha^{\prime} / \beta^{\prime}\right)$, and gamma $(\gamma)$-which differentiate in a strict developmental order (Lee et al. 1999) and exhibit varied expression levels for several genes preferentially expressed in the MBs (Crittenden et al. 1998), suggesting different functional roles. The axons of the $\alpha / \beta$ and the $\alpha^{\prime} / \beta^{\prime}$ MB neurons split into two separate branches, while the axons of the $\gamma \mathrm{MB}$ neurons are unbranched.

Genetic and pharmacological disruptions of the MB neurons that result in anatomical defects or even complete absence of these neurons impair or abolish the learning of odors paired with electric shock reinforcement without grossly perturbing sensorimotor responses to the stimuli (de Belle and Heisenberg 1994; Connolly et al. 1996; Davis 1996; Heisenberg 1998; Wolf et al. 1998). Additionally, various genes associated with learningand memory-related biochemical cascades have elevated levels of expression in MB neurons (Nighorn et al. 1991; Han et al. 1992; Skoulakis et al. 1993; Han et al. 1996; Skoulakis and Davis 1996; Muller 1997; Grotewiel et al. 1998; Roman and Davis 2001). Some of these genes encode molecules that are involved in cyclic AMP (cAMP) signaling: rutabaga (rut) encodes a $\mathrm{Ca}^{2+} /$ calmodulindependent adenylyl cyclase (AC) that converts ATP to cAMP; dunce $(d n c)$ encodes a phosphodiesterase involved in degrading cAMP; and DCO codes for a protein kinase A catalytic subunit. The importance of the cAMP signaling pathway for learning and memory is further supported by disruptions of genes encoding

${ }^{4}$ Corresponding authors.

E-mail dakalal@bcm.tmc.edu; fax (713) 798-8005.

E-mail rdavis@bcm.tmc.edu; fax (713) 798-8005.

Article published online before print. Article and publication date are at http:// www.learnmem.org/cgi/doi/10.1101/lm.221206. other molecules involved in this pathway to produce memorydefective flies; the list of these disruptions has grown to include G-protein subunits, regulatory subunits of protein kinase, and cAMP-responsive transcription factor CREB (Waddell and Quinn 2001; Davis 2005). Other genes that are preferentially expressed in Drosophila MBs include leonardo (leo, 14-3-3), Volado (vol, codes for an $\alpha$-integrin), and fasciclin II (fasII), whereas amnesiac (amn, homologous to a pituitary peptide that activates AC) is expressed preferentially in dorsal paired medial (DPM) neurons that project axons to the MB (Waddell et al. 2000). Mutations in these genes result in learning and memory defects (for review, see Roman and Davis 2001; Davis 2005).

Although the convincing picture that emerges suggests that the Drosophila MBs are an important center for associative learning and memory of olfactory stimuli, the issue of the precise roles for the different neurons comprising the MBs is controversial. Zars et al. (2000a) claimed that restoring rut AC expression specifically in the $\gamma$ neurons of an otherwise rut mutant is sufficient to support normal olfactory learning. In contrast, McGuire et al. (2003) reported that expression of rut in both $\alpha / \beta$ and $\gamma$ neurons is required to obtain complete rescue of the rut learning defect. They further reported that the synaptic output of $\alpha / \beta$ neurons is required for olfactory memory retrieval, but not acquisition or consolidation (McGuire et al. 2001). For the latter experiments, they specifically expressed in these neurons the temperaturesensitive shibire (shi) gene, which encodes a defective dynamin GTPase that dominantly disrupts synaptic transmission at temperatures above $29^{\circ} \mathrm{C}$ quickly and reversibly (Kitamoto 2001). In other studies involving Drosophila long-term olfactory memory, mutants with malformed MBs that were missing $\alpha / \alpha^{\prime}$ axon collaterals have no detectable long-term memory using a spaced training protocol (Pascual and Preat 2001), suggesting that longterm memory forms specifically in the $\alpha / \alpha^{\prime}$ collaterals or that it is retrieved specifically from these branches.

Olfactory learning assays in Drosophila are typically performed by exposing the flies to two odors in succession, one odor 
(the conditioned stimulus, CS+) paired with electric shock pulses (unconditioned stimulus; US) followed by a counter-odor (the CS -) without electric shock. Following training, the flies are presented with the two odors in a T-maze, and their avoidance of the CS+ is computed as a performance index (P.I.). Several variations of this assay have been developed (for review, see Davis 2005), but one variation that may have added to the confusion surrounding the roles of the different neurons of the MBs is the use of different odorants. Studies that have examined the contributions of the different $\mathrm{MB}$ neurons for olfactory learning have used different combinations of odors in the assays. Although most studies have used 4-methylcyclohexanol (MCH) and 3-octanol (OCT) as the CS+ and CS - (Connolly et al. 1996; McGuire et al. 2003; Isabel et al. 2004), some studies have used OCT and benzaldehyde (BEN) (McGuire et al. 2001), or MCH and BEN (Zars et al. 2000a). A recent paper by Keene et al. (2004) describes that flies sense BEN both by the classical olfactory pathway as well as a noncanonical route that involves bitter-sensitive gustatory neurons and pox-neuro (poxn)-affected neurons that are likely on the legs or mouthparts of Drosophila. The question posed by these variations in the use of odorants for olfactory training is whether olfactory associative learning is equally supported irrespective of the choice of odors, or whether the combination of odors used has an effect on the learning that takes place. It is possible, for instance, that different odors are represented differentially among the three classes of $\mathrm{MB}$ neurons. If the different odors are not represented equally by the MB neurons, either because of wiring specificities or differences in activation properties, this could explain some of the discrepancies that are found in the literature regarding the relative importance of the different $\mathrm{MB}$ neurons in olfactory learning.

Expression of rut in the MBs during adulthood has been shown to be sufficient for the rescue of the rut memory impairment, while expression during development is inconsequential to olfactory memory formation (McGuire et al. 2003; Mao et al. 2004). In our study, we examined the effects of restoring rutencoded AC specifically to different neurons of the MBs of rut learning-deficient mutants. In parallel experiments, we drove the $\mathrm{MB}$ neuron-specific expression of a shi transgene to disrupt synaptic transmission at temperatures above $29^{\circ} \mathrm{C}$ and examined the effects on Drosophila learning. Both sets of experiments were performed using the three different odor combinations $(\mathrm{MCH}$ vs. OCT, MCH vs. BEN, and OCT vs. BEN). To our knowledge, this is the first time that experiments using all three odor combinations and using two different experimental manipulations have been used to investigate the roles of the different $\mathrm{MB}$ neurons. Our results demonstrate that although we did not detect any odorspecificity using drivers that express in Drosophila $\gamma$ neurons, a thin core of $\alpha / \beta$ neurons may be differentially involved in learning to specific combinations of odors.

\section{Results}

\section{GAL4 enhancer detector lines express in distinct subsets of Drosophila MB neurons}

Expression patterns of different enhancer detector lines were examined by GAL4-dependent $\beta$-gal expression in adult Drosophila brain. We selected lines that have been identified in previous experiments to have enriched expression within subsets of $\mathrm{MB}$ neurons (Yang et al. 1995; Armstrong et al. 1998; Martin et al. 1998; Zars et al. 2000a,b; McGuire et al. 2001). In addition, we screened numerous new GAL4 lines and identified a driver with enriched expression in the $\gamma$ neurons (NP1131-GAL4). Comparison of GAL4 lines $17 \mathrm{~d}$ and $\mathrm{c} 739$ that were stained for the same amount of time (Fig. 1A,C) showed that although both have
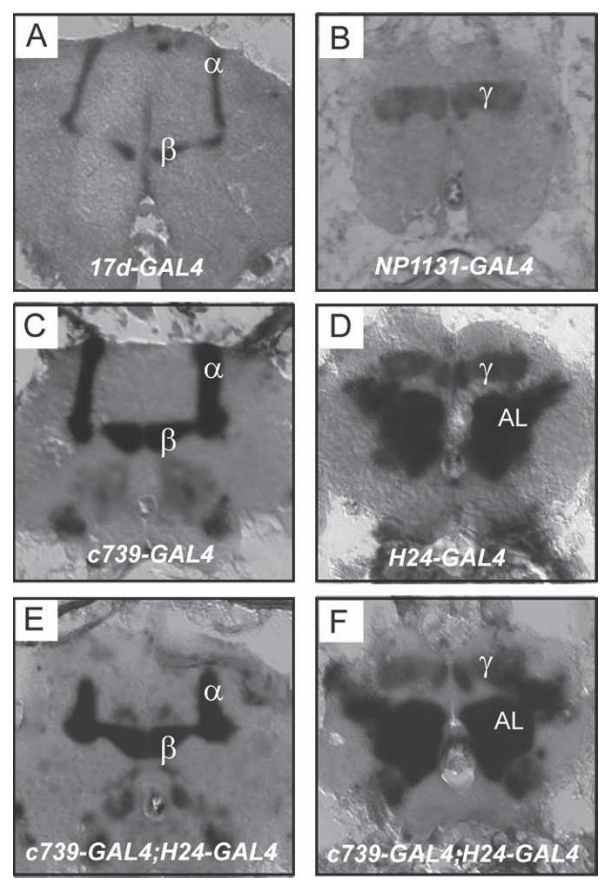

Figure 1. Spatial expression patterns in the brains of flies carrying different GAL4 enhancer detector elements and a UAS-IacZ transposon. Frontal sections through the adult brain were stained for $18 \mathrm{~h}$ in a humidified chamber at $37^{\circ} \mathrm{C}$ for $\beta$-galactosidase activity and examined. Sections at the level of the mushroom body $\alpha / \beta$ and $\gamma$ lobes are shown. $(A, C)$ GAL4 lines $17 d$ and c739 showed expression in the mushroom body $\alpha / \beta$ lobes, although the expression level of $17 d-G A L 4$ was less because this line marks only a subset of $\alpha / \beta$ neurons when compared to $c 739-G A L 4$, which stained the $\alpha / \beta$ lobes more completely. $(B, D)$ GAL4 lines NP1131 and $\mathrm{H} 24$ showed common expression in the $\gamma$ lobes of the mushroom bodies with H24-GAL4 exhibiting robust expression in the antennal lobes $(\mathrm{AL})$ as well. $(E, F)$ Double GAL4 line $\mathrm{C} 739 ; \mathrm{H} 24$ showed expression in both the $\alpha / \beta$ lobes and the $\gamma$ lobes.

expression in the $\mathrm{MB} \alpha / \beta$ lobes, the expression level of $17 d-G A L 4$ is less, and it stains only a subset of $\alpha / \beta$ neurons when compared to $c 739-G A L 4$, which appears to stain the $\alpha / \beta$ lobes more completely. GAL4 lines NP1131 and H24 (Fig. 1B,D) showed common expression in the $\gamma$ neurons of the MBs with line H24-GAL4 additionally exhibiting robust expression in the antennal lobes (ALs) and ellipsoid bodies. Higher expression of the reporter is likewise observed for H24-GAL4, with NP1131-GAL4 showing modest levels of expression in the $\gamma$ neurons but no expression in the ALs. In addition, NP1131-GAL4 expression is observed in a subset of $\alpha^{\prime} / \beta^{\prime}$ neurons that is better detected using GFP (Ito et al. 2003; N.K. Tanaka and K. Ito, unpubl.). For the double GAL4 line c739;H24 (Fig. 1E,F), two representative sections are shown that confirm expression in both the $\alpha / \beta$ and $\gamma$ neurons. Compared to the single GAL4 drivers c739 and H24, the expression levels do not appear to be significantly altered when a combination of the two GAL4 drivers is present in the same fly.

\section{Driving rut expression in $\gamma$ neurons results in partial rescue of the rut learning defect for all odor combinations tested}

Since it is well-established that the MBs are critical for olfactory learning and memory (for review, see Roman and Davis 2001; Waddell and Quinn 2001; Dubnau et al. 2003; Heisenberg 2003; Davis 2005), we wanted to determine whether the neurons comprising the different lobes played specific roles in olfactory learning and memory. We therefore examined the effects of restoring 
rut-encoded AC specifically to different subsets of $\mathrm{MB}$ neurons of rut learningdeficient mutants. GAL4 drivers that express in the $\gamma$ neurons are not numerous; a commonly used line has been $\mathrm{H} 24$ GAL4 (Zars et al. 2000a; McGuire et al. 2003). However, in addition to the $\gamma \mathrm{MB}$ neurons, H24-GAL4 also has robust expression in the ALs (Fig. 1D), and this extraneous expression might affect odor perception that would produce defects in olfactory learning. In fact, the expression of a constitutively activated stimulatory heterotrimeric GTP-binding protein $\alpha$-subunit (Connolly et al. 1996) by H24-GAL4 impairs olfactory avoidance and produces a severe wing phenotype (data not shown). We thus used NP1131GAL4, which shows enriched expression in $\gamma$ neurons (Fig. 1B), in addition to H24-GAL4, to verify the role of the $\gamma$ neurons in olfactory learning.

We first assessed the responses of the different genotypes used in our studies by obtaining $2-5$-d-old flies and testing each genotype for normal odor and shock avoidance (Table 1). Odor avoidance was assayed by measuring the choice of naive flies between odor and fresh air at odor concentrations used during training and testing. For each odorant, a one-way ANOVA with genotype as the main effect was performed, and experiments were comprised of $n \geq 6$ per group. Electric shock avoidance was assayed by measuring the avoidance response of naive flies to $90 \mathrm{~V}$ DC shock. A one-way ANOVA with genotype as the main effect was per-

D
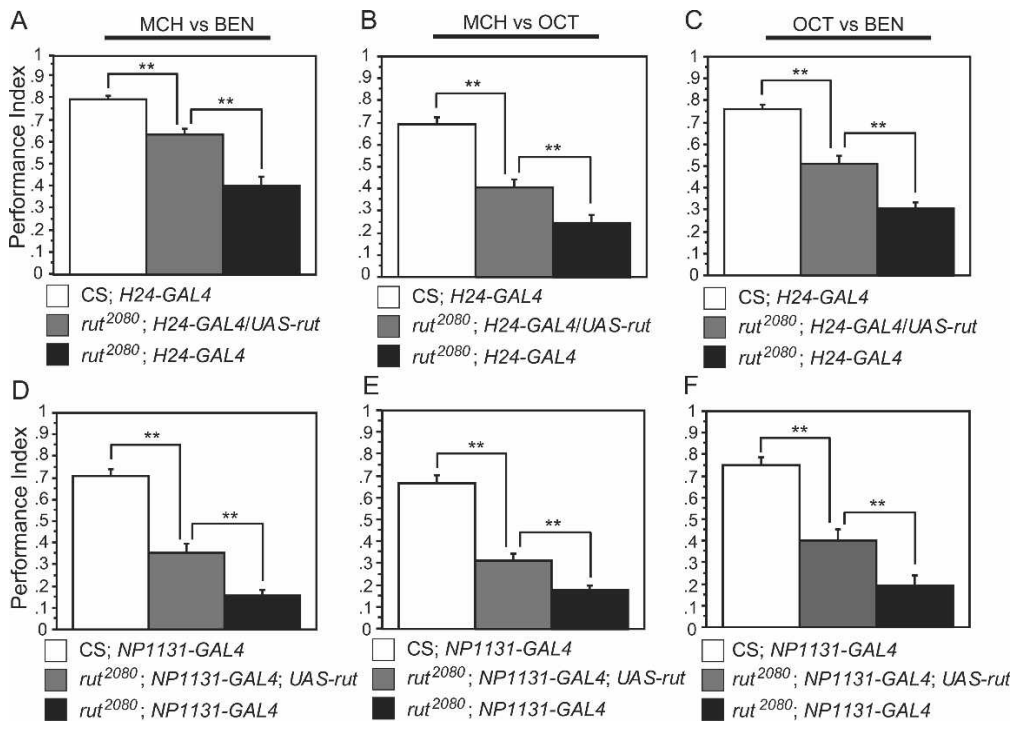

Figure 2. Restoring rut in $\gamma \mathrm{MB}$ neurons produces a partial rescue of the learning-deficiency of rut mutants for all odors tested. Three-minute memory for flies carrying GAL4 drivers that express in $\gamma$ neurons. $(A, B, C)$ Flies bearing $\mathrm{H} 24-\mathrm{GAL} 4$ in combination with the UAS-rut transgene demonstrated significant rescue of rut memory defect over the mutant control group rut ${ }^{2080} ; H 24-G A L 4(P \leq 0.0006)$, but were significantly impaired compared to control flies that were CS; H24-GAL4 for all odor combinations tested $(P \leq 0.0006)$. $n=18$ per group except $n=12$ for the MCH-BEN experiment. $(D, E, F$ Flies of the genotype rut ${ }^{2080}$; NP1131-GAL4; UAS-rut performed significantly better than control flies that were rut ${ }^{2080}$; NP1131-GAL4 ( $\left.P \leq 0.0049\right)$, but were significantly impaired compared to control flies that were CS; NP1131-GAL4 for all odor combinations tested $(P \leq 0.0001)$. $n=18$ per group except $n=17$ for MCH-BEN experiment. This suggests that driving rut in $\gamma$ neurons results in partial rescue of the rut learning defect irrespective of odor combination used. In all experiments, the P.I.'s were subjected to a one-way ANOVA with genotype as the main effect, followed by post hoc analysis with the Bonferroni/Dunn test. Statistical significance is indicated by asterisks $\left({ }^{* *}\right)$.

formed, and experiments were comprised of $n=6$ per group. No significant differences were observed for odor and shock avoidance for the genotypes used in the rut rescue experiments (Table 1).

Table 1. Odor and shock avoidance scores for the different fly stocks used in the rut rescue experiments

\begin{tabular}{|c|c|c|c|c|}
\hline \multirow[b]{2}{*}{ Genotype } & \multicolumn{3}{|c|}{ Odor avoidance } & \multirow[b]{2}{*}{ Shock avoidance } \\
\hline & $\mathrm{MCH}$ & OCT & BEN & \\
\hline $\begin{array}{l}\text { CS; H24-GAL4 } \\
\text { rut } 2080 ; \text { H24-GAL4/UAS-rut } \\
\text { rut }{ }^{2080} ; \text { H24-GAL4 }\end{array}$ & $\begin{array}{l}0.84 \pm 0.06 \\
0.90 \pm 0.01 \\
0.89 \pm 0.02\end{array}$ & $\begin{array}{l}0.78 \pm 0.04 \\
0.74 \pm 0.03 \\
0.78 \pm 0.05\end{array}$ & $\begin{array}{l}0.92 \pm 0.02 \\
0.90 \pm 0.04 \\
0.94 \pm 0.02\end{array}$ & $\begin{array}{l}0.90 \pm 0.03 \\
0.86 \pm 0.04 \\
0.84 \pm 0.04\end{array}$ \\
\hline $\begin{array}{l}\text { CS; NP1131-GAL4 } \\
\text { rut 2080; NP1131-GAL4; UAS-rut } \\
\text { rut 2080; NP1131-GAL4 }\end{array}$ & $\begin{array}{l}0.73 \pm 0.08 \\
0.76 \pm 0.06 \\
0.75 \pm 0.04\end{array}$ & $\begin{array}{l}0.78 \pm 0.04 \\
0.68 \pm 0.06 \\
0.73 \pm 0.04\end{array}$ & $\begin{array}{l}0.73 \pm 0.08 \\
0.76 \pm 0.06 \\
0.75 \pm 0.04\end{array}$ & $\begin{array}{l}0.84 \pm 0.04 \\
0.84 \pm 0.03 \\
0.87 \pm 0.04\end{array}$ \\
\hline $\begin{array}{l}\text { CS; c739-GAL4 } \\
\text { rut 2080; c739-GAL4; UAS-rut } \\
\text { rut }{ }^{2080 ; ~ c 739-G A L 4 ~}\end{array}$ & $\begin{array}{l}0.62 \pm 0.03 \\
0.66 \pm 0.07 \\
0.68 \pm 0.06\end{array}$ & $\begin{array}{l}0.74 \pm 0.03 \\
0.76 \pm 0.06 \\
0.75 \pm 0.04\end{array}$ & $\begin{array}{l}0.82 \pm 0.03 \\
0.80 \pm 0.04 \\
0.82 \pm 0.03\end{array}$ & $\begin{array}{l}0.80 \pm 0.08 \\
0.76 \pm 0.05 \\
0.75 \pm 0.04\end{array}$ \\
\hline $\begin{array}{l}\text { CS; } 17 d-G A L 4 \\
\text { rut } 2080 ; 17 d-G A L 4 ; \text { UAS-rut } \\
\text { rut }^{2080 ;} ; 17 d-G A L 4\end{array}$ & $\begin{array}{l}0.66 \pm 0.03 \\
0.67 \pm 0.04 \\
0.70 \pm 0.05\end{array}$ & $\begin{array}{l}0.66 \pm 0.05 \\
0.55 \pm 0.07 \\
0.60 \pm 0.06\end{array}$ & $\begin{array}{l}0.78 \pm 0.07 \\
0.76 \pm 0.04 \\
0.74 \pm 0.03\end{array}$ & $\begin{array}{l}0.82 \pm 0.08 \\
0.62 \pm 0.06 \\
0.72 \pm 0.08\end{array}$ \\
\hline $\begin{array}{l}\text { CS; c739-GAL4; H24-GAL4 } \\
\text { rut } 2080 ; \text { c739-GAL4; H24-GAL4/UAS-rut } \\
\text { rut }^{2080 ; ~ c 739-G A L 4 ; ~ H 24-G A L 4 ~}\end{array}$ & $\begin{array}{l}0.74 \pm 0.07 \\
0.75 \pm 0.07 \\
0.78 \pm 0.03\end{array}$ & $\begin{array}{l}0.69 \pm 0.07 \\
0.74 \pm 0.03 \\
0.68 \pm 0.02\end{array}$ & $\begin{array}{l}0.85 \pm 0.05 \\
0.82 \pm 0.04 \\
0.82 \pm 0.05\end{array}$ & $\begin{array}{l}0.84 \pm 0.04 \\
0.82 \pm 0.04 \\
0.80 \pm 0.02\end{array}$ \\
\hline
\end{tabular}

Odor avoidance was assayed by measuring the choice of naive flies between odor and fresh air at odor concentrations used during training and testing. Air was bubbled through an odorant:mineral oil mixture. Odor avoidance scores were compared based on the GAL4 driver used and compared with control groups that were comprised of the GAL4 driver crossed with wild-type $w$ flies or the GAL4 driver crossed with the UAS-rut transgene. For each odorant, a one-way ANOVA with genotype as the main effect was performed, and experiments were comprised of $n \geq 6$ per group. No significant differences for odor avoidance were observed $(P \geq 0.1749)$. Electric shock avoidance was assayed by measuring the avoidance response of naive flies to $90 \mathrm{~V} \mathrm{DC}$ shock. A one-way ANOVA with genotype as the main effect did not detect any significant differences $(P \geq 0.0759, n=6$ per group). 
The results in Figure 2 demonstrate that restoring rutencoded AC specifically to the different neurons defined by $\mathrm{H} 24$ GAL4 or NP1131-GAL4 results in a partial rescue of the learning deficiency in rut mutants. This partial rescue was observed for the different odor combinations tested: MCH-BEN (Fig. 2A,D), MCHOCT (Fig. 2B,E), and OCT-BEN (Fig. 2C,F). In each case, flies of the genotype rut ${ }^{2080}$; H24-GAL4/UAS-rut performed significantly better than control flies that were $\mathrm{rut}^{2080} ; \mathrm{H} 24-G A L 4$, but significantly worse than control flies that were CS; H24-GAL4 (Fig. 2A,B,C). Likewise, flies of the genotype rut ${ }^{2080}$; NP1131-GAL4; $U A S$-rut performed significantly better for all odor pairs than control flies that were rut ${ }^{2080}$; NP1131-GAL4, but significantly worse than control flies that were CS; NP1131-GAL4 (Fig. 2D,E,F). It has been shown that expression of rut in the MBs during adulthood is sufficient for the rescue of the rut memory impairment, while expression during development is inconsequential to olfactory memory formation (McGuire et al. 2003; Mao et al. 2004). It has also been established using an inducible gene-switch system that $24 \mathrm{~h}$ of time is sufficient to increase levels of rut so that memory scores are indistinguishable from those of wild-type flies (Mao et al. 2004). Since we used flies that are at least $2 \mathrm{~d}$ old, rut should be at sufficient levels within the spatial expression pattern defined by the GAL4 driver to promote rescue of the rut memory impairment. Our results, therefore, establish that expression of rut in the $\gamma \mathrm{MB}$ neurons defined by either H24-GAL4 or NP1131GAL4 is insufficient to promote complete behavioral rescue, irrespective of the odor combination used. However, the partial
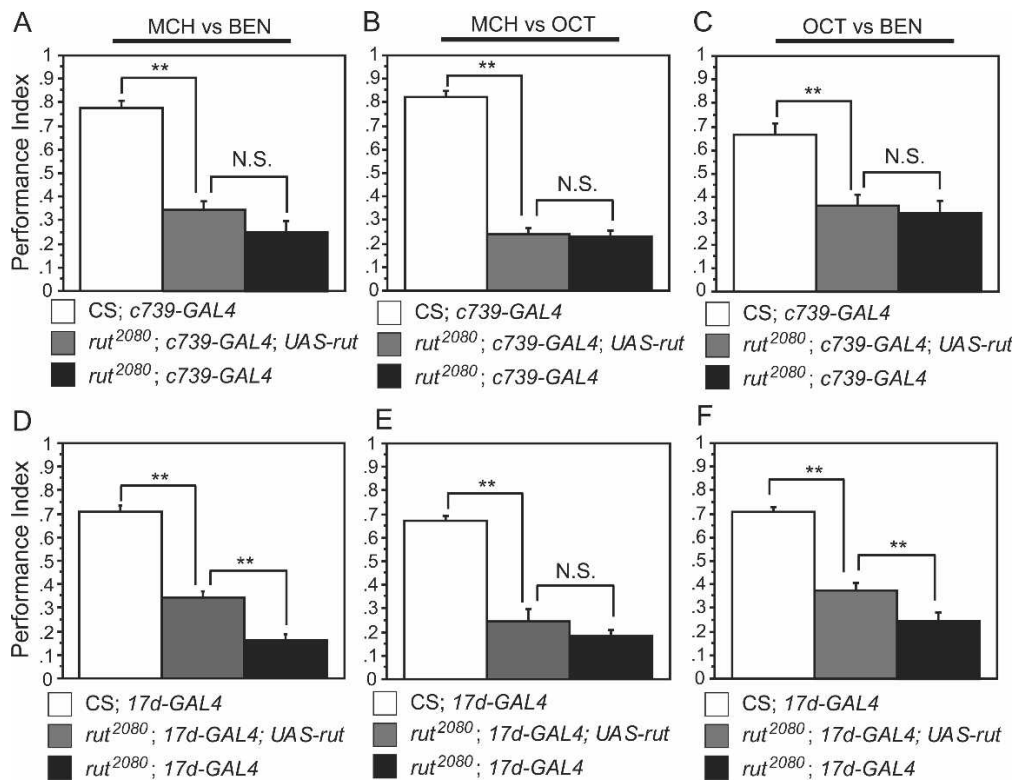

Figure 3. Restoring rut in a subset of $M B \alpha / \beta$ neurons results in partial rescue of the learning deficiency of rut mutants for two odor pairs. Three-minute memory rescue in lines carrying GAL4 drivers that express in $\alpha / \beta$ neurons. $(A, B, C)$ Rut rescue with $c 739-G A L 4$ using different odor combinations. Flies bearing $c 739-G A L 4$ in combination with the UAS-rut transgene demonstrated no significant rescue of rut memory defect over the mutant control group rut ${ }^{2080}$; c739-GAL4 for odor combinations MCH-BEN $(P=0.0886, n=12)$, MCH-OCT $(P=0.7554, n=10)$, and OCT-BEN $(P=0.6218, n=12)$. For all odor combinations tested, rut ${ }^{2080}$; $c 739-G A L 4 ;$ UAS-rut flies performed significantly differently from control flies that were CS; C739-GAL4 $(P<0.0001)$, but were indistinguishable from control flies that were rut ${ }^{2080} ; c 739-G A L 4$. ( $\left.D-F\right)$ Rut rescue with $17 d-G A L 4$, a line that expresses in a subset of $\alpha / \beta$ neurons, using different odor combinations. Flies bearing the GAL4 driver $17 \mathrm{~d}$ in combination with the UAS-rut transgene demonstrated significant rescue of the rut memory defect over the mutant control group rut ${ }^{2080} ; 17 d-G A L 4$ for odor combinations $\mathrm{MCH}-\mathrm{BEN}(P<0.0001, n=24)$ and OCT-BEN $(P=0.0047, n=12)$, but not $\mathrm{MCH}-\mathrm{OCT}(P=0.2577, n=12)$. For all odor combinations tested rut $^{2080} ; 17 d$-GAL4; UAS-rut flies performed significantly more poorly than control flies that were CS; $17 d$-GAL4 $(P<0.0001)$. In all experiments, the P.I.'s were subjected to a one-way ANOVA with genotype as the main effect, followed by post hoc analysis with the Bonferroni/Dunn test. Statistical significance $\left({ }^{* *}\right)$ or nonsignificance (N.S.) is indicated. rescue observed suggests that $\mathrm{AC}$ expression in the $\gamma$ neurons is formed.

\section{Expression of rut and $s h i^{t i l}$ in a subset of $\boldsymbol{\alpha} / \boldsymbol{\beta}$ neurons} edor-pair-specific effect on olfactory learning (McGuire et al. 2001). In contrast, mutan notable difference between these two experiments was the use of combinations of odorants for the learning assay: Mc(2003) used MCH and OCT. Furthermore, rut mutant flies carrythe odor combinations used, we performed rut rescue experiusing these two drivers and the three different odor pairs. ; 739-GAL4; UAS-rut exhibited performance that was indistinguishable from that of $r u t^{2080}$; $c 739-G A L 4$ flies. Surpriswith $17 d-G A L 4$, which targets a subset of $\alpha / \beta$ neurons, conferred partial rescue with the MCH-BEN and OCT-BEN combinations (Fig. 3D,F), but not with the $\mathrm{MCH}-\mathrm{OCT}$ combination (Fig. 3E).

To extend these odor-pair-specific behavioral studies, we used the GAL4 drivers to inactivate synaptic transmission from $\alpha / \beta$ neurons using the dominant and temperature-sensitive $s h i^{t s 1}$ transgene (Kitamoto 2001). We first extended the aforementioned experiments of McGuire et al. (2001) by examining the effects of driving $U A S$-shits ${ }^{\text {ts }}$ using c739-GAL4 for all three odor combinations. Training and testing for 3-min memory was performed entirely at the permissive $\left(25^{\circ} \mathrm{C}\right)$ or restrictive $\left(32^{\circ} \mathrm{C}\right)$ temperatures. In each experiment, we compared the performance of $c 739$ GAL4; UAS-shits ${ }^{\text {ts }}$ flies to control flies bearing just the GAL4 element $(w ; c 739$ GAL4) or the UAS-shits element alone $\left(w ; U A S-s h i^{t s 1}\right)$ at the permissive $\left(25^{\circ} \mathrm{C}\right)$ and restrictive $\left(32^{\circ} \mathrm{C}\right)$ temperatures. For each odor pair, performance of c739GAL4; UAS-shi ${ }^{\text {ts1 }}$ flies at $25^{\circ} \mathrm{C}$ was indistinguishable from that of control flies that were $w$; $c 739-G A L 4$ and $w$; UAS$s h i^{t s 1}$. The c739-GAL4; UAS-shits ${ }^{\text {ts }}$ flies exhibited a partially impaired performance at the restrictive temperature irrespective of the odor pair used for training and testing (Fig. 4A,B,C). This deficit is consistent with previous experiments demonstrating a disruption of 3-min memory when c739-GAL4 drives the expression of $U A S-s h i^{t s 1}$ (McGuire et al. 2001). However, it stands in contrast with our previous results demonstrating

\section{Learning \& Memory}

www.learnmem.org 

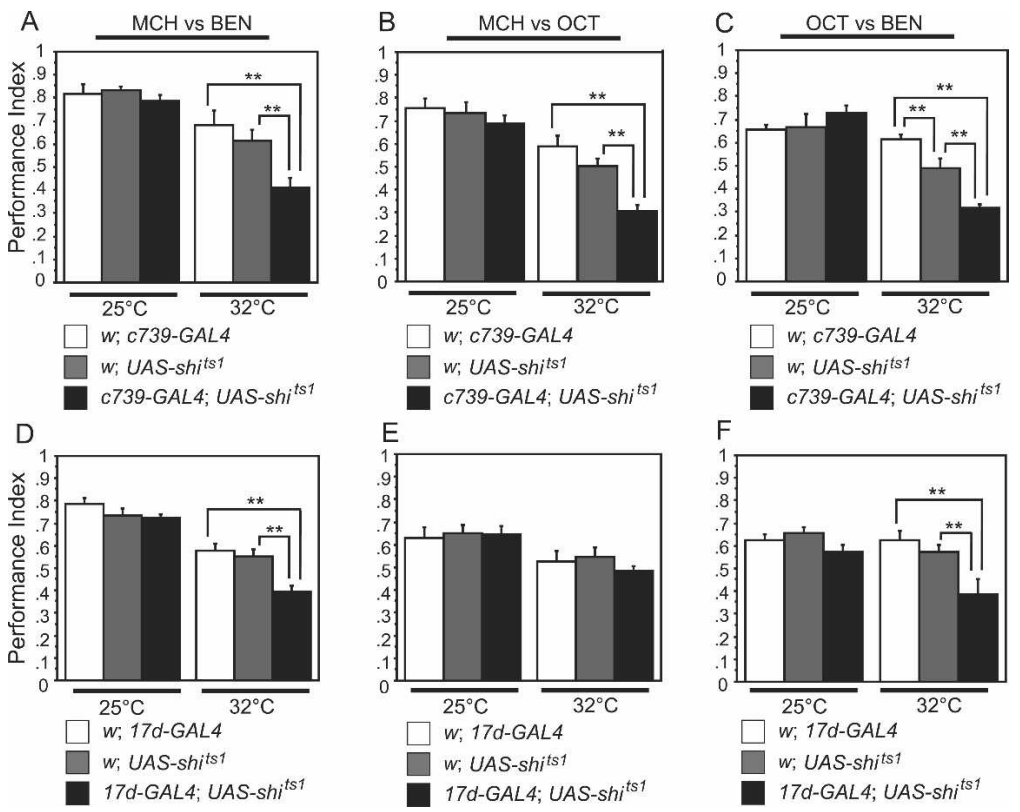

Figure 4. Odor-pair-specific disruption of 3-min memory when $M B$ signaling from $\alpha / \beta$ neurons is blocked. Three-minute memory performance of flies carrying UAS-shits in combination with the GAL4 drivers $\mathrm{C} 739$ or $17 \mathrm{~d}$ with training and testing performed under permissive $\left(25^{\circ} \mathrm{C}\right)$ and restrictive $\left(32^{\circ} \mathrm{C}\right)$ conditions. The different odor combinations used for the experiments are indicated above the graphs: MCH-BEN $(A, D)$, MCH-OCT $(B, E)$, and OCT-BEN $(C, F)$. $(A, B, C)$ Performance of flies of the genotype c739-GAL4; UAS-shits ${ }^{\text {ts }}$ were indistinguishable from controls $w ;$ c739-GAL4 and $w ;$ UAS-shit $i^{\text {ts }}$ at the permissive temperature for MCH-BEN $(P \geq 0.2820, n=6)$, MCH-OCT $(P \geq 0.4691, n=10)$, and OCT$\operatorname{BEN}(P \geq 0.2569, n=6)$, but showed a significant disruption of memory at the restrictive temperature for MCH-BEN $(P \leq 0.0127, n=6)$, MCH-OCT $(P \leq 0.0014, n=6)$, and OCT-BEN $(P \leq 0.0002, n=9)$. $(D-F)$ Performance of flies of the genotype 17d-GAL4; UAS-shit ${ }^{\text {ts }}$ were indistinguishable from controls w; 17d-GAL4 and $w ;$ UAS-shits ${ }^{\text {ts }}$ at the permissive temperature for MCH-BEN $(P \geq 0.0981, n=6)$, MCH-OCT $(P \geq 0.7679, n=10)$, and OCT-BEN $(P \geq 0.0404, n=13)$. Significant disruptions of memory were observed for $17 d$-GAL4; UAS-shits flies at the restrictive temperature when compared to controls for MCH-BEN $(P \leq 0.0008)$ and OCT-BEN $(P \leq 0.0111)$ but not for MCH-OCT $(P \geq 0.2310$, $n=10$ for all). In all experiments, the P.I.'s were subjected to a one-way ANOVA with genotype as the main effect, followed by post hoc analysis with the Bonferroni/Dunn test. Comparisons among the three different genotypes were performed, producing three pairwise planned comparisons per experiment at each of the temperatures. Comparisons were not significant unless indicated by asterisks $\left(^{* *}\right)$.

no rescue of the rut memory defect using c739-GAL4 (Fig. $3 \mathrm{~A}, \mathrm{~B}, \mathrm{C})$. This may be caused simply by the nature of each experiment. The rut rescue experiments involve restoring some level of rut specifically to the neurons defined by the GAL4 drivers. On the other hand, the $s h i^{t s 1}$ experiments involve blocking neurotransmission from these neurons. It is possible that under the conditions described for the experiments (see Materials and Methods), the levels of rut restored to the neurons defined by c739-GAL4 are insufficient to restore performance to levels that are indistinguishable from controls, but that inactivating neurotransmission from these neurons is sufficient to produce significant deficits in performance.

We also assayed 3-min memory for flies bearing the GAL4 element $17 \mathrm{~d}$ along with the $U A S$-shit ${ }^{t s 1}$ transgene. In each experiment, we compared the performance of $17 d-G A L 4$; UAS-shits ${ }^{\text {t1 }}$ flies to control flies bearing only the GAL4 element $(w ; 17 d-G A L 4)$ or the $U A S$-shi ${ }^{t s 1}$ element alone ( $w$; UAS-shits $)$ at the permissive and restrictive temperatures. We observed the same odor-pair-specific effects in these experiments that were observed in the rut rescue experiments involving $17 d-G A L 4$ (Fig. 3). The 17d-GAL4; UAS$s h i^{\text {ts } 1}$ flies showed disruption of 3-min memory at the restrictive temperature using MCH-BEN (Fig. 4D) and OCT-BEN (Fig. 4F) when compared to the $w ; 17 d-G A L 4$ and $w ; U A S-s h i^{t s 1}$ controls. In contrast, no disruption at the restrictive temperature was observed using the odorants $\mathrm{MCH}$ and OCT (Fig. 4E). This is consistent with our previous results with $17 d-G A L 4$ that showed no true for all odor combinations, we repeated these experiments using all three odor combinations. Flies of the genotype rut ${ }^{2080}$; c739-GAL4; H24-GAL4/UAS-rut performed significantly better than control flies that were $r u t^{2080}$; c739-GAL4; H24-GAL4 and had performance scores that were indistinguishable from control flies that were CS; c739-GAL4; H24-GAL4 (Fig. 5). This complete rescue was observed for all odor combinations tested. We cannot determine from these experiments whether this complete rescue occurs by completing a spatial circuit that involves odorant representation in both $\alpha / \beta$ and $\gamma$ neurons, or whether it represents the massed effect of expression in more MB neurons.

\section{$17 d-G A L 4$ expresses in less than half the number of neurons compared to C739-GAL4}

To extend our examination of the expression patterns of the different GAL4 drivers, we visualized the expression patterns at the level of the MB cell body region using antibodies against nuclear-localized GFP and the pan-neural marker ELAV (Robinow et al. 1988). Whole-mount brains were examined under a confocal microscope, and serial sections were taken at $0.5-\mu \mathrm{m}$ intervals. Representative Z-projections of confocal stacks that were generated using ImageJ (Abramoff et al. 2004) are shown in Figure 6A. Only one brain hemisphere is shown since the brains for each group showed overall stereotypy and bilateral symmetry. Each panel was from an individual fly, and expression of GAL4 
Akalal et al.

Table 2. Odor and shock avoidance scores for the different fly stocks used in the $s h i^{\text {ts }}$ experiments

\begin{tabular}{|c|c|c|c|c|c|}
\hline \multirow[b]{2}{*}{ Genotype } & \multirow[b]{2}{*}{ Temperature } & \multicolumn{3}{|c|}{ Odor avoidance } & \multirow[b]{2}{*}{ Shock avoidance } \\
\hline & & $\mathrm{MCH}$ & OCT & BEN & \\
\hline \multirow[t]{2}{*}{ w; c739-GAL4 } & $25^{\circ} \mathrm{C}$ & $0.77 \pm 0.04$ & $0.82 \pm 0.04$ & $0.92 \pm 0.02$ & $0.90 \pm 0.02$ \\
\hline & $32^{\circ} \mathrm{C}$ & $0.79 \pm 0.03$ & $0.68 \pm 0.03$ & $0.88 \pm 0.05$ & $0.78 \pm 0.04$ \\
\hline \multirow[t]{2}{*}{ W; UAS-shi ${ }^{t s 1}$} & $25^{\circ} \mathrm{C}$ & $0.64 \pm 0.05$ & $0.88 \pm 0.03$ & $0.91 \pm 0.03$ & $0.82 \pm 0.03$ \\
\hline & $32^{\circ} \mathrm{C}$ & $0.65 \pm 0.05$ & $0.76 \pm 0.03$ & $0.94 \pm 0.02$ & $0.84 \pm 0.02$ \\
\hline \multirow[t]{2}{*}{ C739-GAL4; UAS-shi ts1 } & $25^{\circ} \mathrm{C}$ & $0.60 \pm 0.05$ & $0.74 \pm 0.05$ & $0.90 \pm 0.03$ & $0.88 \pm 0.02$ \\
\hline & $32^{\circ} \mathrm{C}$ & $0.65 \pm 0.04$ & $0.71 \pm 0.04$ & $0.84 \pm 0.06$ & $0.72 \pm 0.05$ \\
\hline \multirow[t]{2}{*}{$w ; 17 d-G A L 4$} & $25^{\circ} \mathrm{C}$ & $0.72 \pm 0.04$ & $0.91 \pm 0.04$ & $0.88 \pm 0.05$ & $0.80 \pm 0.03$ \\
\hline & $32^{\circ} \mathrm{C}$ & $0.58 \pm 0.07$ & $0.76 \pm 0.02$ & $0.81 \pm 0.06$ & $0.56 \pm 0.08$ \\
\hline \multirow{2}{*}{ w; UAS-shi ${ }^{t s 1}$} & $25^{\circ} \mathrm{C}$ & $0.68 \pm 0.06$ & $0.82 \pm 0.04$ & $0.84 \pm 0.04$ & $0.87 \pm 0.03$ \\
\hline & $32^{\circ} \mathrm{C}$ & $0.62 \pm 0.05$ & $0.76 \pm 0.02$ & $0.90 \pm 0.05$ & $0.62 \pm 0.06$ \\
\hline \multirow[t]{2}{*}{$17 d-G A L 4 ;$ UAS-shi ts1 } & $25^{\circ} \mathrm{C}$ & $0.75 \pm 0.05$ & $0.87 \pm 0.04$ & $0.83 \pm 0.02$ & $0.79 \pm 0.04$ \\
\hline & $32^{\circ} \mathrm{C}$ & $0.44 \pm 0.05$ & $0.74 \pm 0.05$ & $0.87 \pm 0.05$ & $0.59 \pm 0.06$ \\
\hline
\end{tabular}

Odor avoidance was assayed by measuring the choice of naive flies between odor and fresh air at odor concentrations used during training and testing. Air was bubbled through an odorant:mineral oil mixture. For each odorant, a one-way ANOVA with genotype as the main effect was performed for groups tested under similar conditions $\left(25^{\circ} \mathrm{C}\right.$ or $32^{\circ} \mathrm{C} ; n \geq 6$ per group). Post hoc analysis using the Bonferroni/Dunn test indicated that $\mathrm{MCH}$ odor avoidance of flies carrying the UAS-shits ${ }^{\text {tr }}$ transgene with GAL4 driver C739 was lower than the control line w; c739-GAL4 for both temperatures tested $(P \leq 0.0166)$. However, MCH odor avoidance of these flies was not significantly different from the avoidance of $w$; UAS-shits , another control strain used in our experiments $(P \geq 0.5653)$. No other significant differences were found in odor avoidance $(P \geq 0.0266)$. Electric shock avoidance was assayed by measuring the avoidance response of naive flies to $90 \mathrm{~V}$ DC shock. A one-way ANOVA with genotype as the main effect followed by post hoc analysis using the Bonferroni/Dunn test was performed for groups tested under similar conditions $\left(25^{\circ} \mathrm{C}\right.$ or $32^{\circ} \mathrm{C} ; n=6$ per group). No significant differences for shock avoidance were observed $(P \geq 0.0459)$.

drivers was conserved across flies. We counted the number of neurons expressing the GFP reporter after image segmentation and identification of nuclei (Ponomarev and Davis 2003) and found no significant differences $(P \geq 0.4052, n=5)$ between the neuron counts for the left and right MB Kenyon cell (KC) soma region for the different GAL4 drivers examined (Fig. 6B). From our neuron counts, we estimate that $17 d-G A L 4$ is expressed in less than half $(40 \%)$ the number of neurons that express GFP with $c 739-G A L 4$ ( $n=10 \mathrm{KC}$ soma regions from five flies). Further analyses of the confocal Z-series for the two drivers reveal the presence of four clusters of neurons (arrowheads), which probably correspond to their separate lineages. MB cells are derived from four identical neuroblasts via asymmetric divisions through the embryonic, larval, and pupal stages with the $\gamma$ neurons being born before the mid-third instar stage, the $\alpha^{\prime} / \beta^{\prime}$ neurons born between the mid-third instar stage and puparium formation, and the $\alpha / \beta$ neurons born starting at puparium formation (Lee et al. 1999). Comparison of the neuron counts for H24-GAL4 and
NP1131-GAL4 did not reveal any significant difference in the number of labeled neurons $(n=10 \mathrm{KC}$ soma regions from five flies).

\section{Discussion}

Drosophila olfactory learning is typically assayed using olfactory classical conditioning (Tully and Quinn 1985). Using this assay, several memory mutants and the genes involved in olfactory memory formation have been identified (Waddell and Quinn 2001; Dubnau et al. 2003; Heisenberg 2003; Davis 2005). This assay has also been used to investigate the roles of the different MB lobes (Connolly et al. 1996; Zars et al. 2000a; McGuire et al. 2001, 2003; Isabel et al. 2004). In each case, GAL4 drivers that express in distinct lobes of the MBs were used. When G-protein signaling is disrupted using pan-MB GAL4 drivers (238y, c747, and c309) to express a constitutively activated stimulatory heterotrimeric GTP-binding protein $\alpha$-subunit $\left(\mathrm{G}_{\mathrm{s}}{ }^{*}\right)$, associative olfactory learning was reported to be completely abolished (Connolly et al. 1996). Using 201y-GAL4, a line that expresses extensively in the $\gamma$ lobes but only in the narrow core elements of the $\alpha / \beta$ lobes, learning was only reduced by $\sim 50 \%$ (Connolly et al. 1996). In a study that investigated the effects of expressing $s h i^{t s 1}$ using $c 739$ GAL4, an $\alpha / \beta$ driver, and 201y-GAL4, a significant impairment of performance was observed when neurotransmission was transiently inactivated through the $\alpha / \beta$ lobes with only a slight, but nonsignificant, decrease in memory performance observed using 201y-GAL4, suggesting a greater role for $\mathrm{MB} \alpha / \beta$ lobes in olfactory memory (McGuire et al. 2001). Other experiments involving rescue of the rut mutant defect by expressing a wild-type rut cDNA showed that memory was restored to wild-type levels using broad-MB GAL4 drivers (247, c772, and 30y) and the $\gamma$ lobe driver
Figure 5. Complete rescue of the rut learning defect for all odor combinations is achieved when rut is expressed in both $\alpha / \beta$ and $\gamma$ neurons. (A,B,C) Flies of the genotype rut ${ }^{2080} ; c 739-G A L 4 ;$ H24-GAL4) UAS-rut exhibited significantly elevated performance scores compared to the mutant control group rut $^{2080} ;$ c739-GAL4; H24-GAL4 for all odor combinations ( $P<0.0001, n=6$ for all groups). In each case, the performance levels were indistinguishable from control flies of the genotype CS; C739-GAL4, H24-GAL4, indicating complete rescue of the rut learning defect ( $P \geq 0.2853, n=6$ for all groups). In all experiments, the P.I.'s were subjected to a one-way ANOVA with genotype as the main effect, followed by post hoc analysis with the Bonferroni/Dunn test. Statistical significance $\left({ }^{* *}\right)$ or nonsignificance (N.S.) is indicated.

\section{Learning \& Memory} www.learnmem.org 
A
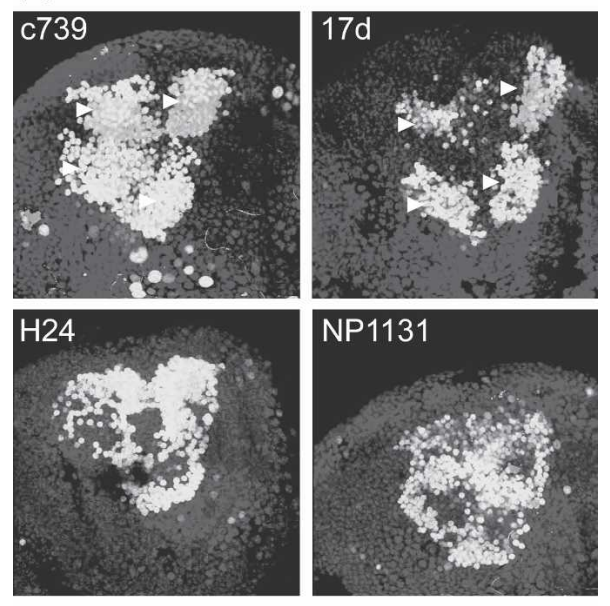

B

\begin{tabular}{|c|c|}
\hline Genotype & $\begin{array}{l}\text { Neuron Counts } \\
\text { (Mean } \pm \text { S.E.) }\end{array}$ \\
\hline C739-GAL4 & $1040 \pm 25(n=10)$ \\
\hline c739-GAL4 left & $1063 \pm 46(n=5)$ \\
\hline c739-GAL4 right & $1017 \pm 22(n=5)$ \\
\hline $17 d-G A L 4$ & $419 \pm 25(n=10)$ \\
\hline 17d-GAL4 left & $445 \pm 30(n=5)$ \\
\hline $17 d-G A L 4$ right & $392 \pm 40(n=5)$ \\
\hline H24-GAL4 & $841 \pm 27(n=10)$ \\
\hline H24-GAL4 left & $824 \pm 38(n=5)$ \\
\hline H24-GAL4 right & $858 \pm 40(n=5)$ \\
\hline NP1131-GAL4 & $868 \pm 41(n=10)$ \\
\hline NP1131-GAL4 left & $865 \pm 56(n=5)$ \\
\hline NP1131-GAL4 right & $871 \pm 67(n=5)$ \\
\hline
\end{tabular}

Figure 6. Neuron counts and GAL4 expression patterns of the different drivers at the level of the $\mathrm{MB}$ cell body region. $(A)$ Expression patterns of 17d-GAL4, C739-GAL4, H24-GAL4, and NP1131-GAL4 in the MB cell body region visualized by crossing the drivers with UAS-GFP:: lacZ.n/s 30.1 flies and doubly stained with anti-ELAV and anti-GFP. Whole-mount brains were examined under a confocal microscope, and serial sections were taken at $0.5-\mu \mathrm{m}$ intervals. Shown are representative projection images of confocal Z-series that were generated using Image) (Abramoff et al. 2004). Brains from five flies per GAL4 line were examined with a representative $M B$ cell body region from individual flies shown for each driver. $(B)$ The number of neurons expressing the GFP reporter was counted after image segmentation and identification of neuronal nuclei (Ponomarev and Davis 2003). Neuron counts for $17 d$-GAL4 were less than half the number of GFP-positive neurons for c739-GAL4 flies $(n=10$ Kenyon cell soma regions from five flies). Neuron counts for H24-GAL4 and NP1131GAL4 were not significantly different from one another $(P=0.5407$, $n=10$ Kenyon cell soma regions from five flies). Brains for each group showed overall stereotypy and bilateral symmetry with no significant differences in neuron counts between the left and right hemispheres $(P \geq 0.4052, n=5$ for each line).

H24-GAL4. However, memory was only partially rescued using 201y-GAL4, and no rescue was observed using the GAL4 drivers $189 \mathrm{y}$ and $17 \mathrm{~d}$, which both express primarily in the $\mathrm{MB} \alpha / \beta$ lobes. This, therefore, suggested a greater role of $\mathrm{MB} \gamma$ lobes in olfactory learning (Zars et al. 2000a). The apparent contradictions among these studies could be due to the fact that in each of these experiments different combinations of odors were used for the olfactory learning assay: MCH-OCT (Connolly et al. 1996), MCHBEN (Zars et al. 2000a), and OCT-BEN (McGuire et al. 2001). In addition, each experiment used a different means of addressing the issue: Connolly et al. (1996) disrupted G-protein signaling by expressing $G \alpha_{s}{ }^{*}$ to abolish learning, McGuire et al. (2001) inactivated neurotransmission using $s h i^{\text {ts } 1}$, and Zars et al. (2000) expressed a rut cDNA in a rut mutant background to rescue the rut learning defect. When McGuire et al. (2003) tried to rescue the rut mutant defect by driving UAS-rut using either $c 739-G A L 4$ or H24-GAL4 alone, the performance of the flies was not significantly different from the rut mutants, but full rescue was observed when a double GAL4 driver c739; H24 was used (McGuire et al. 2003). To resolve the apparent discrepancies and inconsistencies among the different studies, we used all three commonly used odor combinations (MCH-OCT, MCH-BEN, and OCT-BEN) and two different assays, $s h i^{t s 1}$ inactivation of neurotransmission as well as rescue of the rut memory defect, to examine the roles of the different neurons comprising the MB lobes.

Expressing transgenes using the two $\gamma$ lobe GAL4 drivers NP1131 and H24 did not produce any odor-pair-specific learning effects. Using these $\gamma$ drivers to express a rut cDNA in a rut mutant background results in a partial rescue of the learning defect for each of the odor combinations tested (Fig. 2). The $\gamma$ driver of choice has typically been H24-GAL4, but the expression pattern of this driver is not limited to the $\gamma$ neurons. In fact, it expresses very robustly in the ALs (Fig. 1D), and one concern is that this might affect performance scores since the learning assay is based on olfactory cues. The use of NP1131-GAL4 that expresses primarily in the $\gamma$ neurons and a small subset of $\alpha^{\prime} / \beta^{\prime}$ neurons (Fig. 1B) is important to validate the results for $H 24-G A L 4$. A prior study using H24-GAL4 to rescue the rut learning defect resulted in performance scores that were statistically indistinguishable from wild-type flies using $\mathrm{MCH}$ and BEN (Zars et al. 2000a). However, among the different lines that were observed to rescue the rut defect, H24-GAL4 yielded the lowest scores, and another $\gamma$ driver, $201 y$-GAL4, used in the same study failed to rescue the defect (Zars et al. 2000a). Yet another study that used H24-GAL4 to restore a rut cDNA in a rut mutant background failed to produce rescue of the defect using $\mathrm{MCH}$ and OCT as odorants (McGuire et al. 2003). Our data suggest that driving a rut cDNA in $\gamma$ neurons results in rut levels that partially rescue the defect. Although we do not detect any odor-pair-specific learning using the two $\gamma$ drivers we tested, this does not preclude the possibility that when $\gamma$ drivers that express in other subsets of $\gamma$ neurons are discovered, this would remain the case. It is likewise important to note that the three odorants used in our study do not represent the whole repertoire of odors that a fly responds and learns to. Thus, it remains possible that the $\gamma$ neurons labeled by NP1131-GAL4 and H24-GAL4 may still be involved in the odor-pair-specific associative learning of other smells.

Rut rescue using GAL4 drivers that express in the $\alpha / \beta$ neurons reveals some odor-pair-specific effects (Fig. 3). Performance scores for flies carrying c739-GAL4 together with the UAS-rut transgene in a rut mutant background show no rescue of the rut defect for all odor combinations tested. In contrast, driving a rut cDNA using 17d-GAL4 shows partial rescue of the rut defect when MCH-BEN and OCT-BEN are used for the assay but not for MCHOCT. To address the observed contrast in the rescue using the different odor combinations for these two lines, we compared the expression patterns for the two $\alpha / \beta$ drivers. As shown in Figures 1 and 6, the expression level for $c 739-G A L 4$ is greater than $17 d-$ GAL4, and it appears that only a subset of neurons, a thin core, is labeled for $17 d-G A L 4$. Using antibodies raised against glutamate, a further subdivision of the lobes has been described as a slender core of glutamatergic neurons, the $\alpha$ and $\beta$ core neurons ( $\alpha \mathrm{c}$ and $\beta \mathrm{c}$ ), that lie posteriorly and are partly enclosed by the $\alpha$ and $\beta$ neurons (Strausfeld et al. 2003). Whether the core neurons that are marked by $17 d-G A L 4$ correspond to the glutamatergic 
subdivision of the $\alpha / \beta$ lobes remains to be determined. One explanation for the observed odor-pair-specific effects is that $17 \mathrm{~d}$ GAL4 and c739-GAL4 express in nonoverlapping regions of the $\mathrm{MB} \alpha / \beta$ neurons. Our behavioral experiments were performed on flies that were $2-5 \mathrm{~d}$ old, and although our neuron counts make clear that the expression of $c 739-G A L 4$ is broader than $17 d-G A L 4$, we have been unable to confirm whether there is nonoverlapping expression at the $\alpha / \beta$ core. Preliminary data suggest, however, that during this time period, the spatial expression of $17 d$ GAL4 is in the core region, while c739-GAL4 expression is more peripheral with a slight overlap of expression as a ring around this core (I. Sinakevitch, pers. comm.). Additional experiments are needed to extend these observations.

What is the significance of organizing the Drosophila MBs into different lobes, and is there differential representation of odors in the different lobes? Our findings indicate that to answer this question one must take into account the choice of odor pairs used in the olfactory assay. The differential effects we see when using different odor combinations for $17 d-G A L 4$ suggest that even though this driver expresses in a subset of $\alpha / \beta$ neurons, some of these neurons must be important in MCH-BEN and OCT-BEN learning since driving rut using $c 739-G A L 4$, a line that expresses in a greater number of $\alpha / \beta$ neurons, is insufficient to see a partial rescue of the rut learning defect. Clearly, this partial rescue is more than a mass effect of rut-expressing $\mathrm{MB}$ neurons. This raises an important point: It may not be accurate to describe GAL4 driver patterns based solely on lobe-specificity, since different GAL4 drivers may highlight subsets of the neurons comprising individual lobes. The significance of achieving complete rescue when we used the double GAL4 c739; $\mathrm{H} 24$ to express UASrut in a rut mutant background is still unclear. Although it is tempting to speculate that this complete rescue occurs by completing a spatial circuit that involves odorant representation in both $\alpha / \beta$ and $\gamma$ neurons, more experiments need to be performed to investigate whether it may just represent the massed effect of expression in more $\mathrm{MB}$ neurons.

Examination of the odor-evoked activity in Drosophila MB neurons by expressing a green fluorescent protein-based $\mathrm{Ca}^{2+}$ indicator, G-CaMP, have revealed remarkable spatial stereotypy (Wang et al. 2004). In fact, several studies have shown that stereotypical anatomical and functional organization can be found at the different levels of the insect olfactory pathway. Each olfactory receptor neuron (ORN) likely expresses a single olfactory receptor (OR) gene, and ORNs that express the same OR genes converge on a common glomerulus in the $\mathrm{AL}$, resulting in a stereotyped projection pattern (Gao et al. 2000; Vosshall et al. 2000; Scott et al. 2001). A near complete map of ORN connectivity constructed through a systematic survey of Drosophila OR expression has validated the principles of "one neuron-one receptor" and "one glomerulus-one receptor" (Couto et al. 2005). Different odors activate different combinations of ORs (de Bruyne et al. 2001), and individual receptors can mediate both excitatory and inhibitory responses to different odors in the same cell (Hallem et al. 2004). In addition, a topographic organization of the AL has been described wherein ORNs in distinct sensilla types project into distinct regions of the $\mathrm{AL}$ (Couto et al. 2005). At the level of the $\mathrm{MB}$ neuron cell bodies and the calyx, different odors evoked distribution patterns of fluorescence that were odor-specific and conserved across flies, resulting in stereotyped responses for BEN-, MCH-, and OCT-evoked fluorescence activity at both the wide-field and single-neuronal level (Wang et al. 2004). Our results demonstrate an odor-pair-specific effect with 17d-GAL4 using two odor combinations that have BEN as one of the odors. Several studies have indicated that Drosophila processes BEN differently from other odors (Helfand and Carlson 1989; Ayer Jr. and Carlson 1992; Kalidas and Smith 2002). In fact, although surgical removal of the antennae and palps of wild-type flies results in the abolishment of MCH and OCT avoidance, BEN avoidance is only partially affected in both T-maze and arena paradigms, suggesting that BEN is sensed through other nonolfactory pathways (Keene et al. 2004). To eliminate naive odor bias, experiments are usually performed in a counterbalanced design, with half of the flies used in the calculation of the performance index being trained to the first odor and the other half to the second odor. An examination of the half P.I. scores for our experiments does not reveal obvious asymmetries between BEN and the two counter-odors (data not shown). Moreover, the fact that we see the odor-pair-specific effects in both rescue as well as disruption of memory experiments suggests that it is the expression of transgenes in the subset of neurons defined by $17 d-G A L 4$ that confers the odor-pair-specific behavioral phenotypes we observed in this study.

The existence of such stereotypical anatomical and functional organization at the various levels of the Drosophila olfactory pathway may explain the odor-pair-specific rut rescue and $s h i^{t s 1}$-mediated disruption of learning that we observe in our study. The spatial pattern of odor-evoked fluorescence activity for BEN has been reported to occur mostly in the center of the calyx, OCT-evoked activities distribute more laterally and medially, while $\mathrm{MB}$ neurons that displayed fluorescence transients in response to $\mathrm{MCH}$ occur primarily in the top and middle portions of the soma layer (Wang et al. 2004). To investigate whether different lobes of the MBs receive olfactory information from different subsets of AL glomeruli, Tanaka et al. (2004) examined the spatial correlation between $\mathrm{MB}$ neurons and projection neurons (PNs). The MB dendrites of $\gamma$ neurons were found to preferentially occupy the center of the calyx, and although the dendrites of the $\alpha^{\prime} / \beta^{\prime}$ and $\alpha / \beta$ core and surface neurons were more widespread across the calyx, their distribution was slightly nuanced (Tanaka et al. 2004). Based on these observations, we speculate that olfactory learning using different odors is, in part, a function of the relationships between the expression pattern of the GAL4 driver and the degree and pattern of overlap and nonoverlap in the populations of MB neurons that respond to the odor combinations chosen. This is a more complex picture than just having the different odors mapping to distinct lobes of the MBs, and, in fact, a shift toward describing GAL4 drivers based on actual patterns of expression may be more useful than describing them solely on MB lobe-specificity. Ultimately, determining the precise manner by which odors are encoded in the Drosophila brain and how this links to specific behavioral outputs will require careful analyses of the expression patterns of the GAL4 drivers and the representation of the odors in the different MB neurons.

\section{Materials and Methods}

\section{Fly stocks and culture}

Flies were cultured on standard medium at $25^{\circ} \mathrm{C}$ unless the flies were used in the $s h i^{t s 1}$ experiments, in which case they were cultured at $19^{\circ} \mathrm{C}$ to minimize expression of the transgene under the control of the different drivers. Prior to behavioral training, flies were collected in vials and placed overnight in a $25^{\circ} \mathrm{C}$ incubator. Heat shock treatment of adult flies was carried out in vials placed in a $32^{\circ} \mathrm{C}$ water bath for $20-30$ min before training for the $s h i^{t s 1}$ experiments. Canton-S (CS) flies and w(CS10) flies (CS flies carrying the $w 1118$ mutation) served as wild-type controls. In this text, " $w$ " refers to the $w(C S 10)$ allele. The UAS-shits ${ }^{\text {t }}$ flies were those previously used by McGuire et al. (2001) and described in Kitamoto (2001). The UAS-rut flies were those previously used by us (McGuire et al. 2003; Mao et al. 2004) and Zars et al. (2000a,b). These flies have a wild-type $r^{+} t^{+} \mathrm{cDNA}$ under control of the UAS promoter that is inserted onto the third chromosome (Zars et al. 
2000b). The GAL4 lines H24 and 17d were obtained from T. Zars (Theodor Boveri-Institut fur Biowissenschaften, Würzburg, Germany), and c739-GAL4 was from K. Kaiser (Division of Molecular Genetics, University of Glasgow, Scotland). The double GAL4 line c739; H24 was described previously (McGuire et al. 2003).

\section{Task-relevant sensory functions}

Odor avoidance was assayed by measuring the distribution of naive flies between odor and fresh air at the odor concentrations used during training and testing. Air was bubbled through an odorant:mineral oil mixture, and for each odorant, a one-way ANOVA with genotype as the main effect was performed for groups tested under similar conditions. Electric shock avoidance was assayed by measuring the avoidance response of naive flies to $90 \mathrm{~V}$ DC shock. Statistical analyses were performed using STATVIEW. Avoidance indices were subjected to a one-way ANOVA followed by planned comparisons among the relevant groups with a Bonferroni/Dunn post-hoc test. Unless stated otherwise, all data points denoted as "statistically significant" are $P<0.05$.

\section{Memory assay}

Flies were assayed by presenting one of two odors (CS+) with 12 pulses of $90 \mathrm{~V}$ electric shock (US) over $1 \mathrm{~min}$, followed by presentation of the second odor (CS-) with the absence of shock. The three odorants- $\mathrm{MCH}$, OCT, and BEN-were used two at a time (MCH-OCT, MCH-BEN, and OCT-BEN) to represent the CS+ and the CS - in a counterbalanced design, with half of the flies used in the calculation of the performance index being trained to the first odor and the other half to the second odor. Training was performed under dim red light at either $21^{\circ}-25^{\circ} \mathrm{C}$ or $32^{\circ}-35^{\circ} \mathrm{C}$ (for $s h i^{t s 1}$ experiments) and at $60 \%-68 \%$ relative humidity. The electric shock was delivered to the flies through an electrifiable copper grid that served as the floor of the plastic training tubes.

Trained flies were tested in a T-maze in which the two odors were simultaneously presented to the flies. Flies start at the center of the maze and are given 2 min to choose between the two arms of the maze that contained the different odors. Memory was indicated by approaching the CS - and avoiding the CS+. After the 2-min testing period, flies were anesthetized, collected, and counted. Odor concentrations were chosen that yielded 50:50 distributions between the two odors for naive flies. To eliminate odor bias, experiments were performed in a counterbalanced design, with half of the flies used in the calculation of the P.I. being trained to the first odor and the other half to the second odor. The P.I. was calculated by adding the two half P.I.'s and represents the percentage of flies that correctly avoided the CS+ minus the percentage that made the incorrect decision by approaching the CS+. Statistical analyses were performed using STATVIEW. P.I.'s were subjected to a one-way ANOVA followed by planned comparisons among the relevant groups with a Bonferroni/Dunn post hoc test. Unless stated otherwise, all data points denoted as "statistically significant" are $P<0.05$.

\section{Fly histology and microscopy}

To determine the expression pattern of different GAL4 drivers, enhancer detector lines were crossed with a line carrying UASlacZ line and the progeny analyzed. For immunohistochemistry or histochemical staining, $12-\mu \mathrm{m}$ frontal and horizontal sections through the adult fly brain were obtained and examined using a light microscope.

Brains of female progeny flies carrying UAS-GFP::lacZ.nls 30.1 and a GAL4 driver were dissected and double-stained with mouse anti-ELAV and rabbit anti-GFP. This was followed by incubation with Alexa 488 goat anti-mouse and Alexa 594 goat anti-rabbit antibodies. The brain samples were then mounted in $50 \%$ glycerol in $0.1 \%$ PBS. Signals were visualized using a Leica TCS confocal microscope with 488-nm and 594-nm excitation lines with serial sections taken at $0.5-\mu \mathrm{m}$ intervals.

The GFP expression patterns at the level of the MB soma region were examined, and Z-stacks were generated using ImageJ (Abramoff et al. 2004). The number of neurons expressing the GFP reporter was counted after segmentation using an adjust- able-threshold algorithm that segments fluorescently labeled objects contained within three-dimensional images (Ponomarev and Davis 2003). Z-sections were analyzed by " $2 \mathrm{D}$ segmentation of one population." Using this process, neuron counts for each Z-section separated by the average nuclear size were obtained and added up to arrive at an estimate for the total number of neurons per MB body region.

\section{Acknowledgments}

This research was supported by NIH grant NS19904. R.L.D. is the recipient of the R.P. Doherty-Welch Chair in Science at the Baylor College of Medicine. D.-B.G.A. received support from a Jeane B. Kempner Postdoctoral Scholar Award from the University of Texas Medical Branch.

\section{References}

Abramoff, M.D., Magelhaes, P.J., and Ram, S.J. 2004. Image processing with ImageJ. Biophotonics Intl. 11: 36-42.

Armstrong, J.D., de Belle, J.S., Wang, Z., and Kaiser, K. 1998. Metamorphosis of the mushroom bodies; large-scale rearrangements of the neural substrates for associative learning and memory in Drosophila. Learn. Mem. 5: 102-114.

Ayer Jr., R.K. and Carlson, J. 1992. Olfactory physiology in the Drosophila antenna and maxillary palp: acj6 distinguishes two classes of odorant pathways. J. Neurobiol. 23: 965-982.

Connolly, J.B., Roberts, I.J., Armstrong, J.D., Kaiser, K., Forte, M., Tully, T., and O'Kane, C.J. 1996. Associative learning disrupted by impaired Gs signaling in Drosophila mushroom bodies. Science 274: 2104-2107.

Couto, A., Alenius, M., and Dickson, B.J. 2005. Molecular, anatomical, and functional organization of the Drosophila olfactory system. Curr. Biol. 15: 1535-1547.

Crittenden, J.R., Skoulakis, E.M., Han, K.A., Kalderon, D., and Davis, R.L. 1998. Tripartite mushroom body architecture revealed by antigenic markers. Learn. Mem. 5: 38-51.

Davis, R.L. 1996. Physiology and biochemistry of Drosophila learning mutants. Physiol. Rev. 76: 299-317.

. 2005. Olfactory memory formation in Drosophila: From molecular to systems neuroscience. Annu. Rev. Neurosci. 28: 275-302.

de Belle, J.S. and Heisenberg, M. 1994. Associative odor learning in Drosophila abolished by chemical ablation of mushroom bodies. Science 263: 692-695.

de Bruyne, M., Foster, K., and Carlson, J.R. 2001. Odor coding in the Drosophila antenna. Neuron 30: 537-552.

Dubnau, J., Chiang, A.S., and Tully, T. 2003. Neural substrates of memory: From synapse to system. J. Neurobiol. 54: 238-253.

Gao, Q., Yuan, B., and Chess, A. 2000. Convergent projections of Drosophila olfactory neurons to specific glomeruli in the antennal lobe. Nat. Neurosci. 3: 780-785.

Grotewiel, M.S., Beck, C.D., Wu, K.H., Zhu, X.R., and Davis, R.L. 1998. Integrin-mediated short-term memory in Drosophila. Nature 391: $455-460$

Hallem, E.A., Ho, M.G., and Carlson, J.R. 2004. The molecular basis of odor coding in the Drosophila antenna. Cell 117: 965-979.

Han, P.L., Levin, L.R., Reed, R.R., and Davis, R.L. 1992. Preferential expression of the Drosophila rutabaga gene in mushroom bodies, neural centers for learning in insects. Neuron 9: 619-627.

Han, K.A., Millar, N.S., Grotewiel, M.S., and Davis, R.L. 1996. DAMB, a novel dopamine receptor expressed specifically in Drosophila mushroom bodies. Neuron 16: 1127-1135.

Heisenberg, M. 1998. What do the mushroom bodies do for the insect brain? An introduction. Learn. Mem. 5: 1-10.

. 2003. Mushroom body memoir: From maps to models. Nat. Rev. Neurosci. 4: 266-275.

Helfand, S.L. and Carlson, J.R. 1989. Isolation and characterization of an olfactory mutant in Drosophila with a chemically specific defect. Proc. Natl. Acad. Sci. 86: 2908-2912.

Isabel, G., Pascual, A., and Preat, T. 2004. Exclusive consolidated memory phases in Drosophila. Science 304: 1024-1027.

Ito, K., Okada, R., Tanaka, N.K., and Awasaki, T. 2003. Cautionary observations on preparing and interpreting brain images using molecular biology-based staining techniques. Microsc. Res. Tech. 62: $170-186$.

Kalidas, S. and Smith, D.P. 2002. Novel genomic cDNA hybrids produce effective RNA interference in adult Drosophila. Neuron 33: 177-184.

Keene, A.C., Stratmann, M., Keller, A., Perrat, P.N., Vosshall, L.B., and Waddell, S. 2004. Diverse odor-conditioned memories require uniquely timed dorsal paired medial neuron output. Neuron 44: $521-533$. 
Akalal et al.

Kitamoto, T. 2001. Conditional modification of behavior in Drosophila by targeted expression of a temperature-sensitive shibire allele in defined neurons. J. Neurobiol. 47: 81-92.

Lee, T., Lee, A., and Luo, L. 1999. Development of the Drosophila mushroom bodies: Sequential generation of three distinct types of neurons from a neuroblast. Development 126: 4065-4076.

Mao, Z., Roman, G., Zong, L., and Davis, R.L. 2004. Pharmacogenetic rescue in time and space of the rutabaga memory impairment by using Gene-Switch. Proc. Natl. Acad. Sci. 101: 198-203.

Martin, J.R., Ernst, R., and Heisenberg, M. 1998. Mushroom bodies suppress locomotor activity in Drosophila melanogaster. Learn. Mem. 5: $179-191$.

McGuire, S.E., Le, P.T., and Davis, R.L. 2001. The role of Drosophila mushroom body signaling in olfactory memory. Science 293: $1330-1333$.

McGuire, S.E., Le, P.T., Osborn, A.J., Matsumoto, K., and Davis, R.L. 2003. Spatiotemporal rescue of memory dysfunction in Drosophila. Science 302: 1765-1768.

Muller, U. 1997. Neuronal cAMP-dependent protein kinase type II is concentrated in mushroom bodies of Drosophila melanogaster and the honeybee Apis mellifera. J. Neurobiol. 33: 33-44.

Nighorn, A., Healy, M.J., and Davis, R.L. 1991. The cyclic AMP phosphodiesterase encoded by the Drosophila dunce gene is concentrated in the mushroom body neuropil. Neuron 6: 455-467.

Pascual, A. and Preat, T. 2001. Localization of long-term memory within the Drosophila mushroom body. Science 294: 1115-1117.

Ponomarev, A.L. and Davis, R.L. 2003. An adjustable-threshold algorithm for the identification of objects in three-dimensional images. Bioinformatics 19: 1431-1435.

Robinow, S., Campos, A.R., Yao, K.M., and White, K. 1988. The elav gene product of Drosophila, required in neurons, has three RNP consensus motifs. Science 242: 1570-1572.

Roman, G. and Davis, R.L. 2001. Molecular biology and anatomy of Drosophila olfactory associative learning. Bioessays 23: 571-581.

Scott, K., Brady Jr., R., Cravchik, A., Morozov, P., Rzhetsky, A., Zuker, C., and Axel, R. 2001. A chemosensory gene family encoding candidate gustatory and olfactory receptors in Drosophila. Cell 104: 661-673.

Skoulakis, E.M. and Davis, R.L. 1996. Olfactory learning deficits in mutants for leonardo, a Drosophila gene encoding a 14-3-3 protein. Neuron 17: 931-944.
Skoulakis, E.M., Kalderon, D., and Davis, R.L. 1993. Preferential expression in mushroom bodies of the catalytic subunit of protein kinase A and its role in learning and memory. Neuron 11: 197-208.

Strausfeld, N.J., Sinakevitch, I., and Vilinsky, I. 2003. The mushroom bodies of Drosophila melanogaster: An immunocytological and Golgi study of Kenyon cell organization in the calyces and lobes. Microsc. Res. Tech. 62: 151-169.

Tanaka, N.K., Awasaki, T., Shimada, T., and Ito, K. 2004. Integration of chemosensory pathways in the Drosophila second-order olfactory centers. Curr. Biol. 14: 449-457.

Tully, T. and Quinn, W.G. 1985. Classical conditioning and retention in normal and mutant Drosophila melanogaster. J. Comp. Physiol. 157: $263-277$.

Vosshall, L.B., Wong, A.M., and Axel, R. 2000. An olfactory sensory map in the fly brain. Cell 102: 147-159.

Waddell, S. and Quinn, W.G. 2001. Flies, genes, and learning. Annu. Rev. Neurosci. 24: 1283-1309.

Waddell, S., Armstrong, J.D., Kitamoto, T., Kaiser, K., and Quinn, W.G. 2000. The amnesiac gene product is expressed in two neurons in the Drosophila brain that are critical for memory. Cell 103: 805-813.

Wang, Y., Guo, H.F., Pologruto, T.A., Hannan, F., Hakker, I., Svoboda, K., and Zhong, Y. 2004. Stereotyped odor-evoked activity in the mushroom body of Drosophila revealed by green fluorescent protein-based $\mathrm{Ca}^{2+}$ imaging. J. Neurosci. 24: 6507-6514.

Wolf, R., Wittig, T., Liu, L., Wustmann, G., Eyding, D., and Heisenberg, M. 1998. Drosophila mushroom bodies are dispensable for visual, tactile, and motor learning. Learn. Mem. 5: 166-178.

Yang, M.Y., Armstrong, J.D., Vilinsky, I., Strausfeld, N.J., and Kaiser, K. 1995. Subdivision of the Drosophila mushroom bodies by enhancer-trap expression patterns. Neuron 15: 45-54.

Zars, T., Fischer, M., Schulz, R., and Heisenberg, M. 2000a. Localization of a short-term memory in Drosophila. Science 288: 672-675.

Zars, T., Wolf, R., Davis, R., and Heisenberg, M. 2000b. Tissue-specific expression of a type I adenylyl cyclase rescues the rutabaga mutant memory defect: In search of the engram. Learn. Mem. 7: 18-31.

Received February 14, 2006; accepted in revised form June 22, 2006.

\section{Learning \& Memory}




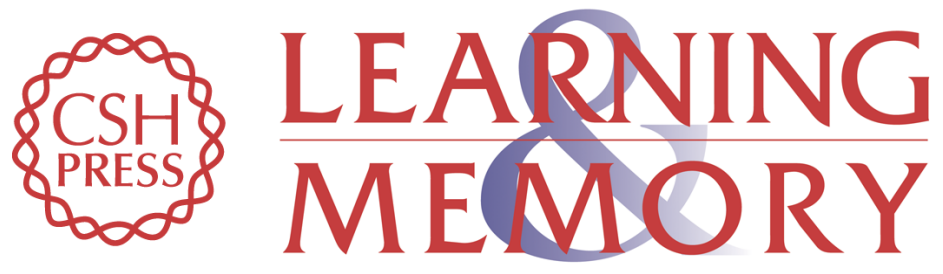

\section{Roles for Drosophila mushroom body neurons in olfactory learning and memory}

David-Benjamin G. Akalal, Curtis F. Wilson, Lin Zong, et al.

Learn. Mem. 2006, 13:

Access the most recent version at doi:10.1101//m.221206

References This article cites 49 articles, 18 of which can be accessed free at:

http://learnmem.cshlp.org/content/13/5/659.full.html\#ref-list-1

License

Email Alerting Receive free email alerts when new articles cite this article - sign up in the box at the Service top right corner of the article or click here. 